\title{
Effet du fractionnement d'engrais organique, d'Urée et du Sulfate de Potassium sur la productivité et la conservation des fruits de tomate au Sud du Bénin
}

\author{
Mensah Armel Clément Goudjo.1, Assogba Komlan Françoise ${ }^{1 .,}$ Azagba Dossou Armand Joël2, \\ Ogoutolou Oscar Romaric Okpèoluwa ${ }^{2}$ et Lucien Guillaume Amadji2 \\ 1 Institut National des Recherches Agricoles du Bénin (INRAB) \\ 2 Faculté des Sciences Agronomiques / Université d'Abomey- Calavi (FSA/UAC). \\ Auteur correspondant E-mail: mensaharmelcg@gmail.com
}

Original submitted in on $20^{\text {th }}$ March 2019. Published online at www.m.elewa.org/journals/ on $30^{\text {th }}$ June 2019 https://dx.doi.org/10.4314/jab.v138i1.5

\section{RESUME}

Objectif : Dans le but d'améliorer la fertilisation de la tomate de plein champ, cette étude a été menée avec hypothèse que la dose d'engrais [1 Matière Organique (MO) + 2 [Urée et Sulfate de Potassium] (EM)] est autant efficace que le fractionnement en 2 pour la MO et 3 pour l'EM.

Méthodologie : Au cours des saisons pluvieuses de 2016 et 2017 , quatre fractionnements (T1 = $1 \mathrm{MO}+2$ $\mathrm{EM}, \mathrm{T} 2=1 \mathrm{MO}+3 \mathrm{EM}, \mathrm{T} 3=2 \mathrm{MO}+2 \mathrm{EM}, \mathrm{T} 4=2 \mathrm{MO}+3 \mathrm{EM}$ ) ont été comparés à un Témoin Absolu (TA) sur la variété de tomate «Padma». Les paramètres de croissance, le rendement et la durée de conservation des fruits sont les variables mesurées. Le rendement du témoin absolu (19 t/ha) est significativement inférieur aux autres modalités T1 (39,22 t/ha); T2 (40,72 t/ha) T3 (37,03 t/ha) et T4 (41,4 t/ha). Les modes de fractionnement n'ont pas influé la durée de conservation des fruits.

Conclusion et application des résultats : Cette étude confirme l'importance de l'Urée et du Sulfate de Potassium dans la fertilisation minérale de la tomate. Les niveaux de rendement obtenus indiquent la nécessité de diffuser l'utilisation de l'Urée et du Sulfate de Potassium en complément minéral aux autres engrais aussi bien en simple ou en double fractionnement.

Mots clés : tomate, fractionnement, matière organique, engrais minéral, rendement, conservation, SudBénin

Effect of fractionation of organic fertilizer, urea and potassium sulphate on the productivity and conservation of tomato fruits in southern Benin

ABSTRACT

Objectives: In order to improve fertilization of tomato in the field, this study was conducted with the assumption that the dose of fertilizer [1 Organic Matter (MO) + 2 [Urea and Potassium Sulphate] (EM)] is as much effective as splitting in 2 for the $\mathrm{MO}$ and 3 for the EM.

Methodology and Results: During the rainy seasons of 2016 and 2017, four fractionations (T1 = $1 \mathrm{MO}+2$ $\mathrm{EM}, \mathrm{T} 2=1 \mathrm{MO}+3 \mathrm{EM}, \mathrm{T} 3=2 \mathrm{MO}+2 \mathrm{EM}, \mathrm{T} 4=2 \mathrm{MO}+3 \mathrm{EM}$ ) were compared to one Absolu control (TA) in a complete four-repeat on the tomato variety "Padma". The tests were conducted in a station on ferralitic soil at INRAB in southern Benin. The variables measured were growth parameters, yield and shelf life of 
fruits under splits. Splits did not have a significant effect on plants height and diameter of tomato. The yield of the absolute control (19 t/ ha) is significantly lower than the other T1 (39.22 t/ ha); T2 (40.72 t / ha) T3 (37.03 t / ha) and T4 (41.4 t/ ha). The fractionation modes did not significantly influence $(P>0.05)$ the quality parameters, in particular the soluble sugar content and the dry matter content. It is the same for the conservation of the fruits where the different modes of fractionation reached $50 \%$ of loss after 20 days in ambient environment.

Conclusion and application of results : This study confirms the importance of Urea and Potassium Sulfate in the mineral fertilization of tomatoes. The yield levels obtained indicate the need to diffuse the use of Urea and Potassium Sulphate as a mineral supplement to other fertilizers both in single and double fractionation.

Keywords : tomato, fractionation, organic matter, mineral fertilizer, productivity, conservation, South Benin

\section{INTRODUCTION}

Au Bénin, la cartographie des sols montre qu'il existe cinq grands types de sols dont le plus dominant est le sol ferralitique (Azontondé, 1991). Ces sols sont caractérisés par un niveau de fertilité très faible, un très faible taux de matière organique (environ 1,33\%), une faible capacité d'échanges cationique, une faible capacité en Phosphore Total et sont moyennement ou fortement acides (Azontondé, 1993; Igué, 2013). De nos jours, l'action combinée de la pression démographique et de la réduction des terres cultivables contraint les producteurs à exploiter les sols ferralitiques desaturés pour la production des cultures maraîchères dont la tomate (Lycopersicon esculentum) de plein champ (Salim, 2014) qui est une culture exigeante en éléments fertilisants (150 à $300 \mathrm{~kg}$ de $\mathrm{N}, 80$ à $200 \mathrm{~kg}$ de $\mathrm{P}_{2} \mathrm{O}_{5}, 200$ à $400 \mathrm{~kg}$ de $\mathrm{K}_{2} \mathrm{O}$ et 30 à $80 \mathrm{~kg}$ de $\mathrm{MgO}$ ) (Bouvier et Jammes, 2012). Déjà en 1990, Gianquinto et Borin ont trouvé qu'un apport de 20 t/ha de fumier est très favorable à l'obtention de rendements élevés de tomate industrielle. Cependant, cet apport doit être complété par une fourniture minérale adéquate de $100 \mathrm{~kg} \mathrm{~N} / \mathrm{ha}+50 \mathrm{~kg} / \mathrm{P}_{2} \mathrm{O}_{5}+140 \mathrm{~kg} / \mathrm{ha} \mathrm{K} 2 \mathrm{O}$. Plus tard en 1995, Elkner et Rumpel ont montré que le fumier seul n'a pas donné satisfaction et ont proposé un apport de fumier à une dose de 40 $\mathrm{t} / \mathrm{ha}$, en plus d'une fumure minérale de $150 \mathrm{~kg} / \mathrm{ha}$ $\mathrm{N}+100 \mathrm{~kg} / \mathrm{ha} \mathrm{P}_{2} \mathrm{O}_{5}+200 \mathrm{~kg} / \mathrm{ha} \mathrm{K}_{2} \mathrm{O}$. Assogba Komlan, (2005) proposait 20 tha de fiente de poulet en apport unique, $400 \mathrm{Kg}$ de NPK, $100 \mathrm{~kg}$ d'urée et $100 \mathrm{Kg}$ de $\mathrm{K}_{2} \mathrm{SO}_{4}$ en deux fractions. Le même auteurs a proposé plus tard en 2016, l'utilisation de la demi-dose pour la matière organique (10 t/ha) et l'engrais minéral NPK (200 $\mathrm{kg} / \mathrm{ha}$ ) et le maintien des doses d'Urée et de Sulfate de Potassium. Malgré cette dernière recommandation, les pratiques de fertilisation généralement observées en milieu paysan au SudBénin, ne tiennent toujours pas compte des doses et périodes d'apports. En effet, des études diagnostiques réalisées dans le sud du Bénin sur la culture de la tomate de plein champ ont montré une grande diversité des pratiques de fertilisation de la culture de tomate. Selon l'enquête menée par Assogba Komlan en 2007, les fientes de poulet constituent la principale source de matière organique utilisée au sud du Bénin à des doses variant entre 26 à 39 t/ha. Pour la fertilisation minérale, deux types d'engrais sont principalement utilisés. II s'agit du NPK avec diverses formules et de l'Urée (Pérrin, 2013). Ces matières organiques et engrais minéraux sont apportés généralement en une fois ou apportés tardivement au cours du cycle végétatif de la culture à des doses très élevées (Dougnon, 2014). Les modes d'apport sont aussi très variables et ne participent pas à une bonne utilisation des éléments fertilisants. Les engrais sont épandus à la volée sans enfouissement dans le sol. Ces différentes pratiques de fertilisation combinées aux caractéristiques physico-chimiques défavorables des sols ferralitiques conduisent au lessivage, au ruissèlement ou à la volatilisation des éléments fertilisants apportés dont les conséquences agronomiques sont : i) le faible rendement (8 t/ha) de la tomate enregistré en milieu producteur (FAO, 2016); ii) la faible qualité des fruits et iii) la courte durée de conservation des fruits. C'est donc pour montrer la faiblesse de ces pratiques de 
fertilisation que cette étude a été initée pour évaluer l'effet du fractionnement d'engrais d'engrais organique (fiente de poulet), de l'urée et

\section{MATERIEL ET METHODES}

Présentation du milieu d'étude: L'étude a été conduite au cours des saisons pluvieuses de 2016 et 2017 sur le site expérimental du Sous-Programme Cultures Maraîchères de l'Institut National des Recherches Agricoles du Bénin (INRAB) situé à Abomey Calavi au sud du Bénin. Les caractéristiques du Sulfate de Potassium sur la productivité et la durée de conservation des fruits de tomate au Sud du Bénin.

chimiques du sol en 2016 et en 2017 avant la mise en place des essais sont présentées dans le tableau 1. Les données pluviométriques collectées pendant les deux années d'expérimentation, au niveau du site de recherche, sont présentées dans la figure 1.

Tableau 1: Composition en éléments chimiques du sol avant expérimentation

\begin{tabular}{c|c|c|c|c|c|c|c}
\hline & & \multicolumn{6}{|c}{ Eléments dosés } \\
\hline Année & Horizon & $\mathbf{C ~ ( \% )}$ & $\mathbf{N}(\%)$ & $\mathbf{C} / \mathbf{N}$ & $\mathbf{M} .0 .(\%)$ & $\begin{array}{c}\text { K ech. } \\
(\mathbf{m e ́ q} / 100 \mathrm{~g})\end{array}$ & $\begin{array}{c}\mathbf{P} \text { ass } \\
\text { (ppm) }\end{array}$ \\
\hline 2016 & \multirow{2}{*}{$0-30 \mathrm{~cm}$} & 0,48 & 0,06 & 8,44 & 0,83 & 0,63 & 73,22 \\
\cline { 1 - 7 } & 0,58 & 0,05 & 8,14 & 0,79 & 0,18 & 64,25 \\
\hline
\end{tabular}

(LSSEE, 2016 et 2017)

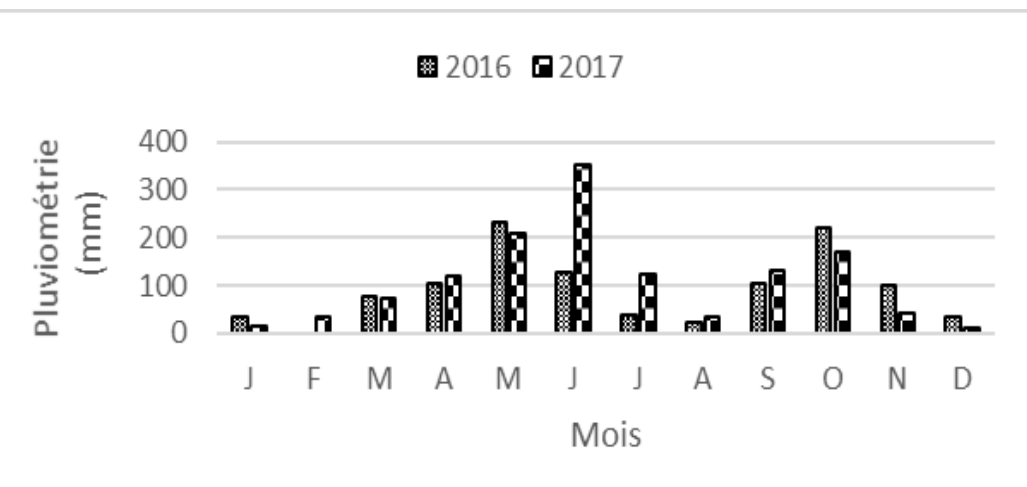

Figure 1 : Variation pluviométrique enregistrée au cours de la période d'étude

Matériel et méthodes : Le matériel végétal utilisé est la variété de tomate Padma 108-F1, résistante au flétrissement bactérien. Les types d'engrais utilisés dans le cadre de l'étude sont :

- $\quad$ Engrais organique : fiente de poulet ;

- $\quad$ Engrais simples : l'Urée $(46 \%$ de $N)$ et le

Sulfate de Potassium $\left(50 \%\right.$ de $\left.\mathrm{K}_{2} \mathrm{O}\right)$;

- $\quad$ Engrais complexe : $\mathrm{N}_{14} \mathrm{P}_{23} \mathrm{~K}_{14} \mathrm{~S}_{5} \mathrm{~B}_{1}$.

Doses testées et périodes d'apport

\section{- Engrais organique}

Pour l'engrais organique, la Dose Initialement Recommandée (DIR) est de 10 tha en un apport unique. Dans le cadre de cette étude, cette dose a été fractionnée en deux apports :

- $\quad 1 / 2$ DIR à 1 SAR (Semaine Après Repiquage)

\begin{abstract}
- $\quad \frac{1}{2}$ DIR à 5 SAR.
- Engrais minéraux

Pour les engrais minéraux, la DIR pour l'Urée et le Sulfate de Potassium en mélange est de $100 \mathrm{Kg} / \mathrm{ha}$ pour chacun de ces types d'engrais en deux apports identiques respectivement au 4 ème et 6ème SAR. Cette dose a été fractionnée en trois apports non identiques qui se présentent ainsi qu'il suit :

$\begin{array}{ll}\text { - } & 1 / 4 \text { de la DIR à la 4ème SAR; } \\ \text { - } & 1 / 2 \text { de la DIR à la } 6 \text { ème SAR; } \\ \text { - } & 1 / 4 \text { de la DIR à la } 8 \text { ème SAR. }\end{array}$

Ainsi, les traitements comparés sont récapitulés dans le tableau 2. A ces traitements, s'est ajouté un témoin absolu (sans aucun apport).
\end{abstract}


Tableau 2. Traitements mis en comparaison

\begin{tabular}{|c|c|c|}
\hline \multirow{2}{*}{$\begin{array}{l}\text { Fractionnement de } \\
\text { l'engrais organique }\end{array}$} & \multicolumn{2}{|c|}{ Fractionnement des doses d'urée et de $\mathrm{K}_{2} \mathrm{SO}_{4}$} \\
\hline & $\begin{array}{c}\text { Simple fractionnement } \\
\text { (Deux apports) } 50+50 \mathrm{Kg} / \mathrm{ha}\end{array}$ & $\begin{array}{c}\text { Double fractionnement } \\
\text { (Trois apports) } 25+50+25 \mathrm{Kg} / \mathrm{ha}\end{array}$ \\
\hline $\begin{array}{l}1 \text { apport d'engrais } \\
\text { organique (10 T/ha) }\end{array}$ & $\begin{array}{c}1 \text { apport de } \mathrm{MO}+2 \text { apports de EM } \\
\mathrm{T} 1\end{array}$ & $\begin{array}{c}1 \text { apport de } \mathrm{MO}+3 \text { apports de EM } \\
\mathrm{T2}\end{array}$ \\
\hline $\begin{array}{l}2 \text { apports d'engrais } \\
\text { organique }(5+5 T / h a)\end{array}$ & $\begin{array}{c}2 \text { apports de } \mathrm{MO}+2 \text { apports de EM } \\
\mathrm{T} 3\end{array}$ & $\begin{array}{c}2 \text { apports de } \mathrm{MO}+3 \text { apports de EM } \\
\text { T4 }\end{array}$ \\
\hline
\end{tabular}

MO : Matière Organique ; EM : Engrais Minéral.

$\mathrm{NB}$ : Un apport de $\mathrm{N}_{14} \mathrm{P}_{23} \mathrm{~K}_{14} \mathrm{~S}_{5} \mathrm{~B}_{1}$ a été apporté à $2 \mathrm{SAR}$ aux traitements $\mathrm{T} 1$ à T4

Dispositif expérimental: Ces traitements ont été installés dans un dispositif expérimental de Bloc Aléatoire Complet en 4 répétitions sur des parcelles élémentaires de 12,8 $\mathrm{m}^{2}$ (3,2 $\left.\mathrm{m} \times 4 \mathrm{~m}\right)$ constituées de quatre lignes de $4 \mathrm{~m}$ de long. Les allées entre les parcelles élémentaires dans un bloc sont de 0,50 m et de $1 \mathrm{~m}$ entre deux blocs. Les plants ont été repiqués après trois semaines de pépinière. L'écartement observé est de 0,80 m entre les lignes et 0,50 m sur la ligne à raison d'un plant par poquet, soit une densité de 25000 plants à l'hectare.

\section{Données collectées}

Paramètres de croissance: Les paramètres de croissance évalués dans le cadre des essais ont été la hauteur des plants et le diamètre au collet. Les mesures ont été prises sur dix plants spécifiquement sur cinq plants dans les deux lignes centrales. La collecte de ces données s'était réalisée de façon hebdomadaire et a démarré deux semaines après le repiquage. Au total, huit observations ont été faites pour ces deux paramètres.

Composantes de rendement: Les principaux éléments mesurés pour l'évaluation du rendement sont : le nombre de plants récoltés, le nombre de fruits récoltés par plant et le poids total des fruits. Le rendement net en fruits de tomate (RNF) a été calculé suivant la formule :

$$
\operatorname{RNF}(\mathrm{T} / \mathrm{Ha})=\frac{\text { Poids total fruits récoltés }(\mathrm{kg}) * 10000 \mathrm{~m}^{2}}{\text { Superficie utile }\left(4,8 \mathrm{~m}^{2}\right) * 1000 \mathrm{~kg}}
$$

Qualité des fruits récoltés : Trois paramètres ont été mesurés sur les plants de tomates pour évaluer l'effet des différentes techniques de fertilisation sur la qualité des fruits de tomate. II s'agit de :

- $\quad$ La Matière Sèche Soluble (MSS) : Cinq fruits ont été choisis par parcelle. Après la récolte, les fruits, sont déposés en milieu ambiant au laboratoire jusqu'à leur mûrissement totale. La mesure de la MSS a été prise à l'aide d'un réfractomètre de marque Hand-Held Refractometers (Model 7530L/7531L/7533L.

- La teneur en matière sèche: Elle a été déterminée suivant la méthode AOAC (AOAC, 2002). Les fruits frais après être nettoyés, découpés et pesés, ont été mis à l'étuve à $105^{\circ} \mathrm{C}$ pendant 48 heures. Les fruits secs sont pesés à la sortie de l'étuve après un passage dans un dessiccateur pour leur refroidissement. Le Taux de Matière Sèche (TMS) est calculé sur la base de la formule :

TMS $(\%)=\frac{100 * \text { Poids fruit sec }(g)}{\text { Poids fruit frais }(g)}$

La durée de conservation des fruits : En fonction des différentes techniques de fractionnement, 40 fruits à raison de dix fruits par répétition ont été mis en conservation en milieu ambiant à l'air libre. Les fruits de tomate ont été étalés sans être superposés à même le sol au laboratoire. La durée de conservation des fruits a été suivie pour les différents traitements. Tous les cinq jours, les fruits pourris au niveau de chaque traitement sont retirés du lot. Les dates correspondant à $25 \%$, $50 \%$ et $75 \%$ de pourriture ont été observées par traitement.

Analyses et traitements statistiques: Pour tester l'effet des traitements sur les paramètres du rendement de la tomate, les modèles linéaires à effets mixtes ont été réalisés avec comme facteurs fixes le traitement et la période (année) et comme facteur aléatoire le bloc. La fonction Imer du package ImerTest a été utilisée pour établir les modèles. Des statistiques descriptives (moyennes, erreurs types) ont été calculées et ont permis de réaliser des graphes, histogrammes/tableaux présentant les différents paramètres de croissance, du rendement suivant les périodes par traitement. Pour tester l'effet des traitements sur la conservation des 
fruits de tomate, la non normalité (test de shapiro-wilk) des données a conduit à réaliser le test non paramétrique de kruskall-wallis. Le test de corrélation linéaire de Pearson a été aussi effectué pour tester la corrélation entre la durée de conservation et la quantité

\section{RESULTATS}

Effet du fractionnement des engrais organiques et minéraux sur les paramètres de croissance

Effet des traitements sur la hauteur et le diamètre au collet des plants de tomate : Les fractionnements d'engrais organiques de l'Urée et du Sulfate de de fruits conservés. Des courbes montrant la tendance évolutive de la quantité des fruits conservés ont été aussi réalisées. Toutes les analyses statistiques ont été réalisées dans le logiciel $R$ version 3.5.1 ( $R$ Core Team, 2018).

Potassium n'ont pas d'effet significatif sur la hauteur $(P$ $>0,05)$ et le diamètre au collet $(P>0,05)$ des plants de tomate. Cependant, le diamètre au collet (Figure 2) et la hauteur des plants de tomate (Figure 3 ) évoluent significativement en fonction du temps $(P=0,000)$.

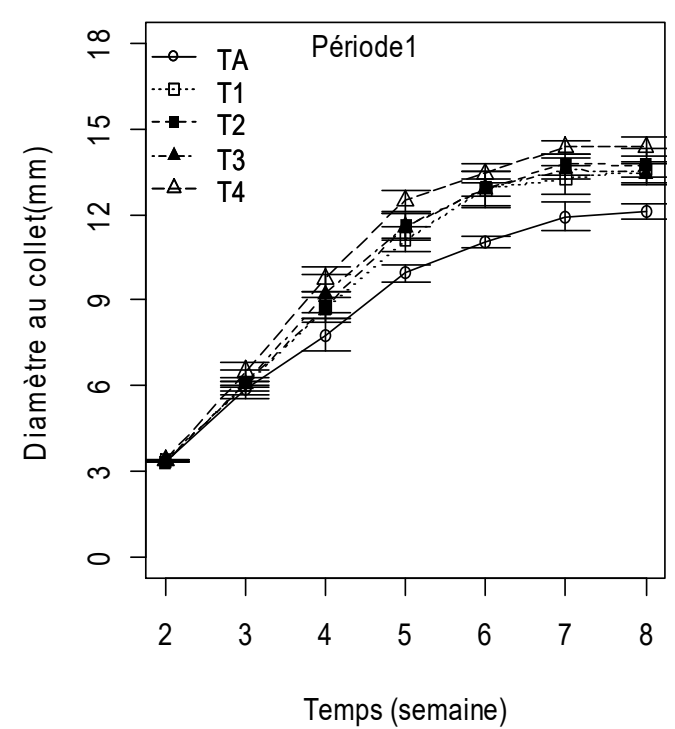

Année 2016

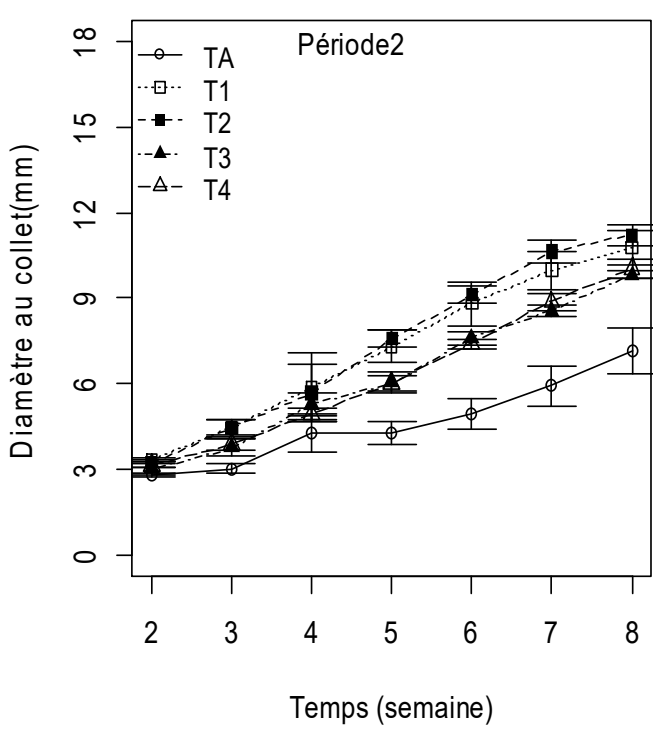

Année 2017

Figure 1 : Evolution du diamètre au collet des plants de tomate par période suivant les fractionnements

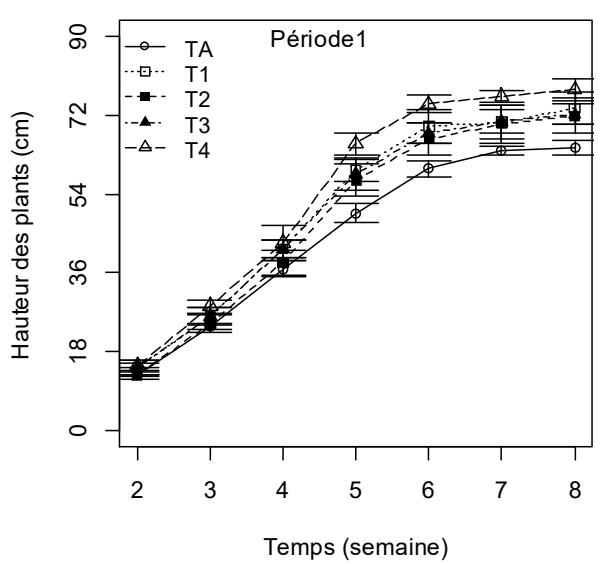

Année 2016

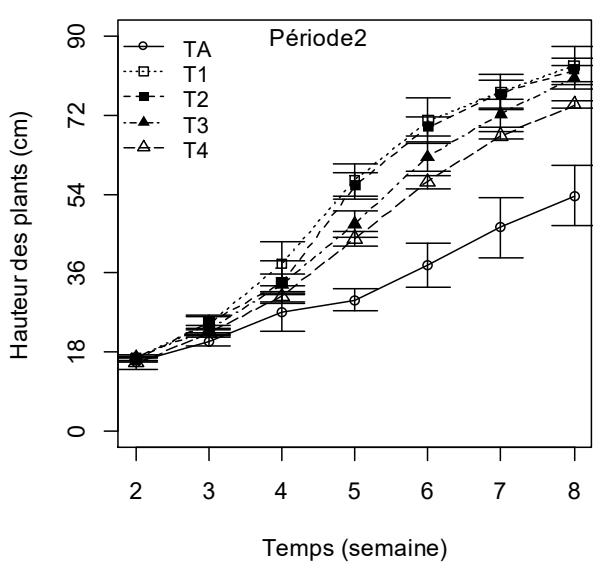

Année 2017

Légende : $\mathrm{TA}=$ Témoin Absolu, $\mathrm{T} 1=1$ apport de $\mathrm{MO}+2$ apports de EM, $\mathrm{T} 2=1$ apport de $\mathrm{MO}+3$ apports de EM, T3= 2 apports de $\mathrm{MO}+2$ apports de EM, T4 = 2 apports de MO +3 apports de EM

Figure 3 : Evolution de la hauteur des plants de tomate par période suivant les fractionnements 
Effet du fractionnement des engrais organiques et minéraux sur les composantes du rendement de la tomate: Le nombre moyen de fruits de tomate par plant a varié suivant les traitements sur les deux années d'étude. Au cours de la première année, le nombre moyen de fruits de tomate par plant n'est pas statistiquement différent entre les traitements $\mathrm{T} 1$ - un apport de matière organique et deux fractionnements d'engrais minéraux, T2 - engrais minéral fractionné en trois apports, T3 - engrais organiques et minéraux fractionnés en deux apports, T4 - engrais organiques et minéraux fractionnés en trois apports (Figure 4a). Au cours de la deuxième année par contre, seul le traitement T2 a obtenu un nombre de fruits statistiquement plus important que celui du témoin absolu (Figure 4a). Pour les deux années d'étude, aucune différence significative n'a été enregistrée au niveau des traitements avec fractionnement d'engrais organique, de l'Urée et du Sulfate de Potassium (T2, T3 et T4) en termes de rendement. Ces fractionnements sont statistiquement identiques à l'apport unique de matière organique et aux deux fractionnements d'Urée et du Sulfate de Potassium (T1) mais statistiquement différents du témoin absolu $(p=0,000)$. En première année d'étude, c'est le traitement (T4) avec deux et trois fractionnements respectivement d'engrais organiques d'Urée et du Sulfate de Potassium qui a donné le meilleur rendement $(41,4 \mathrm{t} / \mathrm{ha})$. En deuxième année (Figure 4b), c'est le traitement (T2) avec un apport de matière organique et trois fractionnements d'Urée et du Sulfate de Potassium qui a donné le meilleur niveau de rendement $(40,72 \mathrm{t} / \mathrm{ha})$.
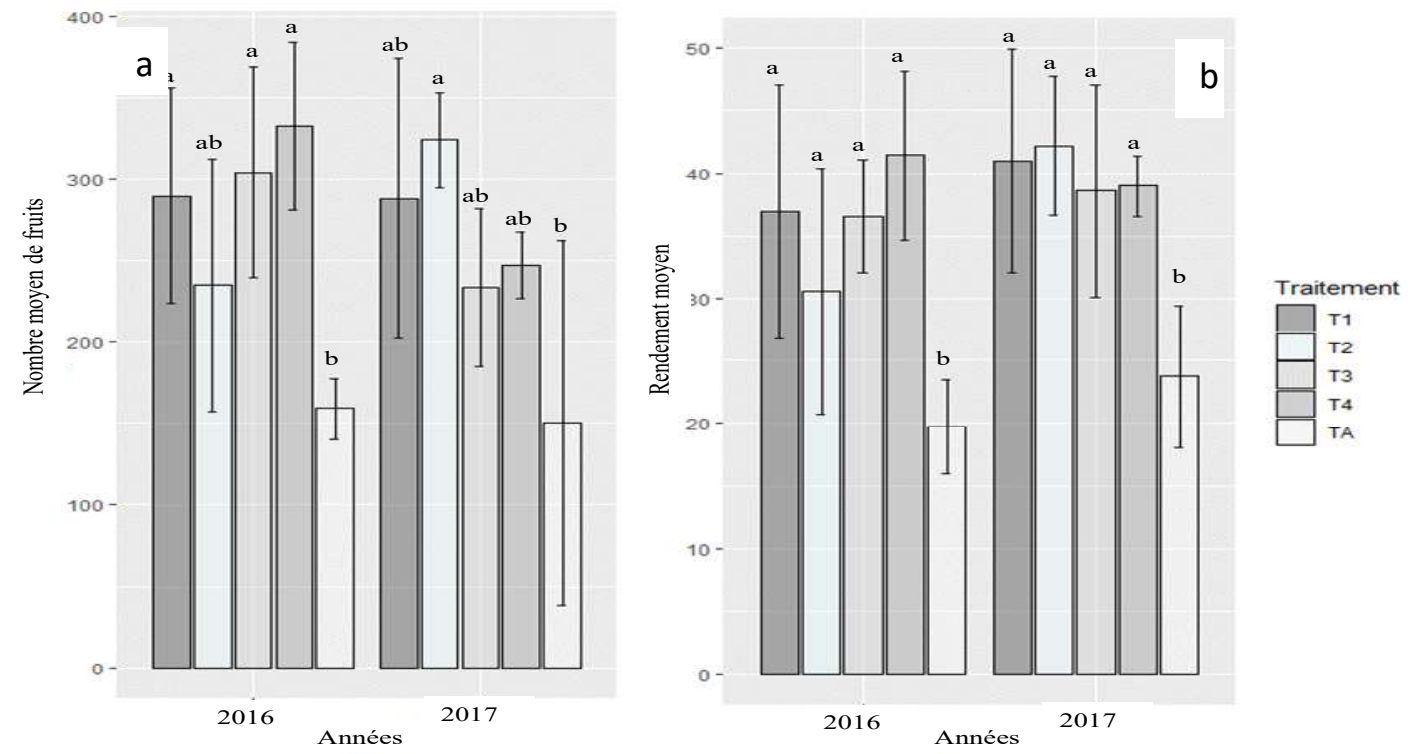

Légende : TA = Témoin Absolu, T1= 1 apport de $\mathrm{MO}+2$ apports de EM, T2 = 1 apport de MO +3 apports de EM, T3= 2 apports de $\mathrm{MO}+2$ apports de EM, T4 $=2$ apports de $\mathrm{MO}+3$ apports de EM

Figure 4 : Variation du nombre de fruits (a) et du rendement moyen de tomate (b) par année suivant les traitements

Effet du fractionnement des engrais organiques et minéraux sur la qualité des fruits de tomate: Effet sur la teneur en matière sèche soluble ( ${ }^{\circ}$ Brix) et le taux de matière sèche $(\%)$ : Les fractionnements d'engrais organique, d'Urée et du Sulfate de Potassium n'ont montré de différence significative sur la teneur en matière sèche soluble $(P>0,05)$ de même que sur le taux de matière sèche $(P>0,05)$ des fruits de tomate (Tableau 3). 
Tableau 3 : Matière sèche soluble et matière sèche suivant les années en fonction des fractionnements

\begin{tabular}{|c|c|c|c|}
\hline Année & Traitements & Matière sèche soluble ( $\left.{ }^{\circ} \mathrm{Brix}\right)$ & $\begin{array}{c}\text { Taux de Matière } \\
\text { Sèche }(\%)\end{array}$ \\
\hline \multirow{5}{*}{2016} & $\mathrm{T1}$ & $4,17^{a} \pm 0,07$ & $3,81^{a} \pm 0,82$ \\
\hline & $\mathrm{T} 2$ & $4,42^{\mathrm{a}} \pm 0,11$ & $4,46^{a} \pm 0,20$ \\
\hline & T3 & $4,13^{a} \pm 0,08$ & $4,55^{a} \pm 0,23$ \\
\hline & $\mathrm{T} 4$ & $4,38^{a} \pm 0,08$ & $3,55^{a} \pm 0,45$ \\
\hline & TA & $4,00^{\mathrm{a}} \pm 0,18$ & $4,42^{\mathrm{a}} \pm 0,17$ \\
\hline \multirow{5}{*}{2017} & T1 & $4,30^{a} \pm 0,16$ & $3,69^{a} \pm 0,20$ \\
\hline & $\mathrm{T} 2$ & $4,28^{a} \pm 0,11$ & $4,22^{\mathrm{a}} \pm 0,14$ \\
\hline & T3 & $4,19^{a} \pm 0,12$ & $3,84^{a} \pm 0,25$ \\
\hline & $\mathrm{T} 4$ & $4,22^{\mathrm{a}} \pm 0,10$ & $3,84^{a} \pm 0,11$ \\
\hline & TA & $4,29^{a} \pm 0,20$ & $3,83^{a} \pm 0,30$ \\
\hline
\end{tabular}

Les mêmes lettres en colonne rapportent les moyennes qui ne sont pas statistiquement différentes à $5 \%$.

Légende : TA = Témoin Absolu, T1 = 1 apport de $\mathrm{MO}+2$ apports de EM, T2 = 1 apport de MO +3 apports de EM, $\mathrm{T} 3=2$ apports de $\mathrm{MO}+2$ apports de EM, T4 = 2 apports de MO +3 apports de EM

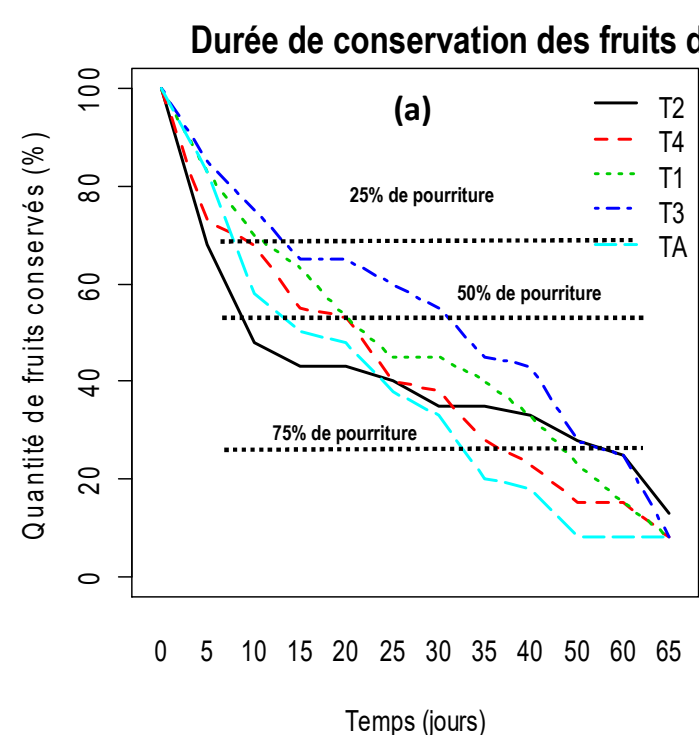

Année 2016

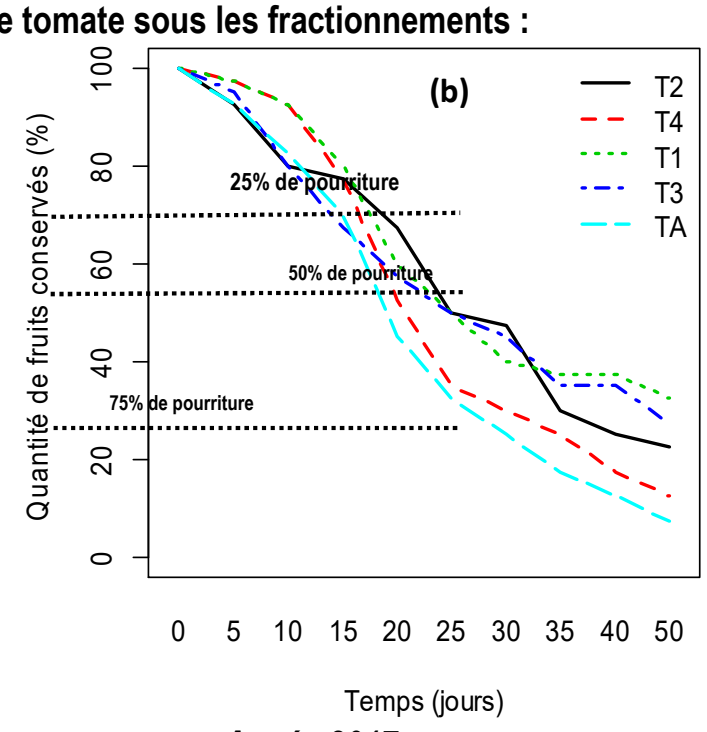

Année 2017

Légende : TA = Témoin Absolu, T1 $=1$ apport de $M O+2$ apports de EM, T2 $=1$ apport de $M O+3$ apports de EM, T3= 2 apports de $\mathrm{MO}+2$ apports de EM, T4 $=2$ apports de $\mathrm{MO}+3$ apports de EM)

Figure 5 : Cinétique de pourriture des fruits de tomate en fonction du temps en 2016 (a) et en 2017 (b) suivant les traitements

Les variations dans la durée de conservation des fruits de tomate sont présentées dans la figure 5 . II ressort de l'analyse de cette figure, que l'évolution des pertes de fruit de tomate au cours des deux années de conservation, a suivi la même tendance pour l'ensemble des pratiques de fertilisation. En première année, les fruits du Témoin Absolu ont atteint 25\%, $50 \%$ et $75 \%$ de pourriture respectivement au 10 ème, $15^{\text {ème }}$ et 20 ème jour de conservation. Quelle que soit l'année, les $75 \%$ de perte en fruit ont été atteints au niveau du T4 (2 apports de MO + 3 apports de EM) entre 30 et 35 jours de conservation. 


\section{DISCUSSION}

II ressort de nos résultats, qu'en plein champ, l'apport unique ou fractionné d'engrais organique combiné à deux ou trois apports d'Urée et de Sulfate de Potassium, n'influence pas différemment la hauteur des plants de la variété «Padma». Les travaux de Koala 0. et Bélem J. (2018) ont mis en évidence sous abri antiinsectes pour la même variété de tomate, l'effet positif de l'application toutes les 3 semaines du NPK et de I'Urée sur la hauteur des plants. En effet, sous abri, la différence de hauteur significative enregistrée serait due à l'effet d'ombrage du filet anti-insectes comme l'ont montré les travaux de Mensah (2016), Gogo (2014) et Djidji (2010). En ce qui concerne le diamètre au collet des plants, nos résultats se rapprochent des travaux de Mpika (2015) qui ont montré que l'apport de fertilisant azoté n'a eu aucun effet significatif sur le diamètre moyen au collet aussi bien de variétés " locale " qu'améliorée " Mongal». Les mêmes tendances ont été observées avec les travaux de Kitabala (2016) lorsque ces derniers ont évalué l'effet de différentes doses de compost sur la production et la rentabilité de la tomate dans les conditions de Kolwezi au Congo. En ce qui concerne le rendement, ceux issus de nos travaux sont supérieurs à ceux obtenus au niveau national ( $8 \mathrm{t} / \mathrm{ha}$ ) et sont également supérieurs à ceux généralement obtenus sur les sols ferralitiques au Sud Bénin (6 t/ha) selon les statistiques de la DSAIMAEP (2016). Ces faibles rendements s'expliquent par la non application de l'Urée en mélange avec le Sulfate de Potassium en culture de tomate de plein champ en milieu paysan. En effet, les diagnostics effectués en milieu paysan au Sud Bénin, ont révélé que les fientes de poulet (Assogba Komlan, 2007), le NPK et l'Urée sont les principales sources de matière organique et d'engrais minéraux utilisés pour la fertilisation de la tomate de plein champ (Dougnon 2014; Pérrin, 2013; Assogba Komlan en 2007). Nos résultats viennent corroborer aussi ceux de Masome et Kazemi (2014) qui ont montré le rôle de l'Urée et du Potassium dans l'accroissement de la production de tomate aussi bien pour les variétés améliorées que

\section{CONCLUSION}

Cette étude vient donc répondre à la faiblesse des modes d'apports des engrais organiques et minéraux de plein champ basé uniquement sur l'utilisation de fientes, du NPK et de l'Urée à des périodes non recommandées au Sud-Bénin. L'étude a montré que lorsque les apports et fractionnements sont effectués aux périodes indiquées, $75 \%$ des fruits de la variété de traditionnelles. Ils sont également similaires à ceux de Mpika (2015) qui a montré que la production est maximale pour un fractionnement en deux apports de l'azote combiné au Potassium pour une variété améliorée de tomate. Si Koala O. et Bélem J. (2018) ont montré que sous abri "anti-insectes", l'application de NPK et d'Urée toutes les deux semaines augmentait les rendements de $51 \%$ par rapport à l'application conventionnelle (une seule application pour NPK et deux applications pour l'Urée), aucune différence significative n'a été obtenue entre le fractionnement d'engrais organique, l'Urée et le Sulfate de Potassium $(2 \mathrm{MO}+2 \mathrm{EM}$ ou $2 \mathrm{MO}+3 \mathrm{EM})$ en plein champ dans nos conditions expérimentales et l'apport unique d'engrais organique combiné à deux apports d'Urée et de Sulfate de Potassium (1 MO + 2 EM). Cette similarité entre ces différentes techniques de fractionnement pourrait être due au fait que tous ces traitements ont reçu l'engrais complexe $\mathrm{N}_{14} \mathrm{P}_{23} \mathrm{~K}_{14} \mathrm{~S}_{5} \mathrm{~B}_{1}$ comme fumure minérale de fond sauf le témoin. Toutefois, ces apports ont permis de doubler le rendement en tomate fruit comparé au témoin sans fertilisation avec un rendement de 19 t/ha. Dans nos conditions expérimentales, les différents fractionnements n'ont eu aucun effet sur les teneurs en sucres solubles et le taux de matière sèche contrairement aux résultats de Koala 0 . et Bélem J. (2018) qui ont montré que sous abri "anti-insectes", pour la même variété de tomate, le fractionnement en 6 applications avec une fréquence d'application de 2 semaines, augmente la teneur en matière sèche soluble. Les durées de conservation enregistrées dans le cadre de cette étude sous les différents factionnements indiquent qu'entre 5 et 15 jours, les fruits étalés à l'air libre en milieu ambiant sans superposition ont atteint $25 \%$ de pourriture. Le traitement T4 qui fractionne d'avantage a atteint les $75 \%$ de perte autour de 30 jours de conservation. Ces résultats sont en adéquation avec ceux de Aïsso, 2016; qui ont montré que les fruits de la variété de tomate «Padma», ne se conservent pas longtemps.

tomate Padma sans superposition, peuvent être conservés en milieu ambiant pendant 30 jours. Bien qu'au stade actuel de l'étude, le simple fractionnement des doses d'Urée et de Sulfate de Potassium soit aussi performant du point de vue agronomique que le double fractionnement, les niveaux de rendements obtenus aussi bien pour 1 apport de $\mathrm{MO}+2$ apports de EM 
(37,0 à 39,22 t/ha); 1 apport de $M O+3$ apports de EM (30,5 à 40,72 t/ha), 2 apports de MO + 2 apports de EM (36,5 à 37,03 t/ha) et 2 apports de $\mathrm{MO}+3$ apports de $\operatorname{EM}(37,84$ à 41,4 t/ha), indiquent la nécessité d'amener

\section{REFERENCES BIBLIOGRAPHIQUES}

Aïsso R.C. B., Assogba Komlan F., Aissi M.V., Mensah C. A., Soumanou M. M., 2016. Profils morphologique, sensoriel et durée de conservation de nouvelles variétés de tomates introduites au Sud-Bénin. Spécial hors-série $n^{\circ} 2$ décembre 2016, Science et technique, Sciences naturelles et agronomie. 260p.

Assogba Komlan F., 2007. Valorisation des déchets agro-industriels en agriculture urbaine dans le Sud du Bénin: Diagnostic, évaluation et perspectives. Thèse de doctorat, URF des Sciences de la Terre et des Ressources Minières de l'Université de Cocody, Abidjan, Côte d'Ivoire, $140 \mathrm{p}$.

Assogba Komlan F., Sikirou R., Azagba J. 2013. Comment réussir la culture de la tomate en toute saison : Cas des régions urbaines et périurbaines $\mathrm{du}$

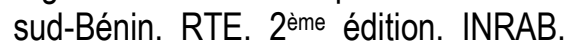
$58 \mathrm{p}$.

Azontondé H. A., 1991. Propriétés physiques et hydrauliques des sols du Bénin. In Proceedings of the Niamey: Soil Water Balance in the Sudano Sahelian Zone, 1991. IAHS. no. $199: 250-258$.

Azontondé A. 1993. Dégradation et restauration des terres de barre (sols ferralitiques faiblement désaturés argilo-sableux) au Bénin. Cah. Orstom, série. Pédologie. vol. XXVIII, N²: 217-226.

Bouvier E. et Jammes D. 2012. Fertilisation en Maraîchage. Les sols vivant bio. Matières organiques Fiche $\mathrm{N}^{\circ} 8$. Maison des Agriculteurs - 22 rue Henri Pontier 13626 Aix-en-Provence, France, $10 \mathrm{p}$.

Djidji A. H., Zohouri G. P., Fondio L., Nzi J. C. Et N. C. Kouamé, 2010. Effet de l'abri sur le comportement de la tomate (Solanum lycopersicum L.) en saison pluvieuse dans le Sud de la Côte-d'Ivoire. Journal of Applied Biosciences 25: 1557 - 1564 ISSN 1997-5902

Dougnon T. V., Bankole H. S., Klotoe J. R., Legonou les producteurs à intégrer l'apport complémentaire de l'urée et de Sulfate dans la production de tomate de plein champ.

M., Dougnon J. T., Adjehidadie T. A. Et Boko M. 2014. Digestion en anaérobiose des fientes de poulets : impacts sur la qualité microbiologique des feuilles de Solanum macrocarpon Linn (Solanaceae) cultivé au Bénin. International Journal of Innovation and Applied Studies, 5(3) : 241-253

DSA/MAEP 2016. Statistiques de production agricoles au Bénin, camapagne 2016

Elkner K., Rumpel J., 1995. Effect of crop rotation and fertilization on quality of processing tomatoes. Acta-Agrobotanica., 48:(2), 17-25.

Gianquinto-G; Borin-M., 1990. Effect of organic and mineral fertilizer application and soil type on the growth and yield of processing tomatoes (Lycopersicon esculentum Mill.). Rivista-di-Agronomia. 24 (4), 339-348.

Gogo Elisha O. Thibaud Martin, Mwanarusi Saidi, Francis M. Itulya and Mathieu Ngouajio : Microclimate Modification Using Ecofriendly Nets for High-quality Tomato Transplant Production by Small-scale Farmers in East Africa, Journal of Hortitechnolgy, Research Reports, june 2012, 22(3). 7p.

Igue A. M., Saidou A., Adjanohoun A., Ezui G., Attiogbe P., Kpagbin G., Gotoechan-Hodonou H., Youl S., T. Pare, Balogoun I., Ouedraogo J., Dossa E., Mando A., Sogbedji J. M., 2013. Evaluation de la fertilité des sols au Sud et au Centre du Bénin, BRAB, Numéro spécial Fertilité du maïs -12-23 pp.

Kitabala Misonga A.Tshala Upite J. Kalenda M. A., Tshijika Ikatalo M., Mufind et $M$. Kayakez 2016. Effets de différentes doses de compost sur la production et la rentabilité de la tomate (Lycopersicon esculentum Mill) dans la ville de Kolwezi, Province du Lualaba (RD Congo). http://dx.doi.org/10.4314/jab.v102i1.1 
Koala O., Bélem J. 2018. Effects of Split Mineral Fertilizer Applications on the Growth and Productivity of Three Tomato Varieties (Lycopersicon esculentum Mill.) in Rainy Season Cropping. DOI https://doi.org/10.1007/978-3-31958792-9_9 Pp 139-160

Masome H., Kazemi S., 2014. Effects of ammonium sulphate and urea fertilizers on the growth and yield of tomato. Journal of Novel Applied Sciences, 3 (2): 148-150.

Mensah A. C. G., S. Simon, F. Assogba Komlan, L. A., T. Martin et M. Ngouajio (2016): Intensification de la culture de tomate sous abri couvert de filet anti-insectes en région chaude et humide du SudBénin. Communication SIST 2016 Ouagadougou, Publié dans Science et Technique, Sciences Naturelles et agronomies, Spécial hors série $n^{\circ} 2-$ Décembre 2016 - ISSN 1011-6028.

Mpika J., Attibayeba, Makoundou A., Minani D., 2015. Influence d'un apport fractionné en potassium et en azote sur la croissance et le rendement de trois variétés de tomate de la zone périurbaine de Brazzaville en République du Congo, Journal of Applied Biosciences 94:8789 $-8800,11-13 p p$.

Nurtika N., Hidayat A., (1992). Effect of time of nitrogen and potassium fertilizer application on growth and yield of tomato TW variety on wet season. Buletin PenelitianHortikultura (Indonesia). (1992). v. 23(3) p. 94-100. Received 1996.

Pérrin A. 2013. Evaluation environnementale des systèmes agricoles urbains en Afrique de l'Ouest : Implications de la diversité des pratiques et de la variabilité des émissions d'azote dans l'Analyse du Cycle de Vie de la tomate au Bénin. Sciences agricoles. 2013. <NNT : 2013AGPT0080>.

Salim S., 2014. La gestion intégrée de la fertilité des sols (GIFS), Almanach 2014, Congo, 1$3 p$. 